\title{
THE TREND OF IONOSPHERIC TOTAL ELECTRON CONTENT NEAR THE EQUATOR
}

\author{
${ }^{1}$ Shehu, M. U. , ${ }^{2}$ Said, R.S. and ${ }^{3}$ Okoro, E.C. \\ ${ }^{1}$ Shehu Idris College of Health Sciences and Technology Makarfi, P.M.B. 1050, Kaduna State Nigeria. \\ ${ }^{2}$ Department of Physics Bayero University Kano P.M.B 3011, Kano Nigeria \\ ${ }^{3}$ Department of Physics and Astronomy University of Nigeria, Nsukka \\ "Corresponding author: mushehuikara@gmail.com
}

\begin{abstract}
The lonospheric Total Electron Content (TEC) data measured simultaneously by analyzing dual-frequency signals of the Global Position System (GPS) in two northern Nigeria equatorial stations: Ahmadu Bello University Zaria (ABUZ) with geographic latitude $\left(11.16^{\circ} \mathrm{N}\right)$ and Longitude $\left(7.65^{\circ}\right.$ E) and Birnin Kebbi Polytechnic (BKFP) with geographic latitude $\left(12.46^{\circ} \mathrm{N}\right)$ and Longitude $\left(4.20^{\circ} \mathrm{E}\right)$ during the year $2012\left(R_{\mathrm{z}}=57.58\right)$ was used to study the diurnal, monthly and seasonal variation of equatorial ionosphere. The result shows that the mean TEC varies from a minimum at 0600 hrs LT to a peak value at about 1500 to 1700 hrs LT and then decreases. The seasonal variation in TEC maximizes during the equinox months followed by winter and is minimum during the summer months.

Key Words: Ionospheric Total Electron Content, Equatorial Stations, Diurnal, Monthly, Seasonal, Variation
\end{abstract}

INTRODUCTION

The earth's ionosphere forms an interface between the atmosphere and space. It is a partially ionized gas that enclosed the earth extends from a height of $50 \mathrm{Km}$ to more than $1000 \mathrm{Km}$ (Kelly). It is very important because of its influence on the passage of radio wave.

The ionosphere is highly dynamical and complicated in structure; consisting of interconnected part of physical system, and plays a significant role in long distance radio communications in HF band (Dabas et al., 2006, Matsushita and Wallace, 1967). The ionosphere is affected by solar activity, sun-earth relative position and interaction among the ionosphere, magnetosphere plus thermosphere etc. (Hong et al., 2008). The variability in the equatorial ionosphere is due to large-scope of electrodynamics actions related with the equatorial electro jet (EEJ) current, plasma fountain, equatorial ionization anomaly (EIA). The equatorial and low latitude ionosphere is characterized in terms of latitudinal of ionization by a depression at the magnetic equator and crests at about $\pm 17^{\circ}$ magnetic latitude, and crest is affected by various geophysical condition (Appleton 1946; Kumar 2009) a prominent attribute which is referred to as the equatorial ionization anomaly (EIA) (Olwendo; et al 2012)etc. This EIA is attributed to the equatorial plasma being moved up by upward $E \times B$ vertical drifts due to eastward electric field, there after diffuse down along the magnetic field line to higher latitudes under the influence of gravitational force (Sanjay and Singh 2009; Bhuyan and Borah, 2007). The overall process is known as the "fountain effect". However the diurnal randomness in the variation of TEC specifically during noon time to predawn hours which is of serious concern in navigation and forecasting require more precise estimation of the ionospheric delay error in the navigation signal due to the free electrons and ions in the ionosphere. (Unnikrishnan and Ravidram 2010; Rao et al., 2009; 2010) The variation in GPS TEC have been study extensively by a number of workers (Chauhan and Singh, 2010; Rao et al., 2006, Bolaji et al 2012; Adewale et al 2012; Rabiu et al, 2007) is recent years due to the availability of Signals under all-weather condition on regional as well as global basis, (Komjathy et al., 1998). The GPS is a satellite based positioning system widely used for navigation, relative positioning and time transfers. The system has three segments - the Space Segment, the Control Segment and the User Segment (the GPS receivers). GPS space segment consists of constellation of 24 satellite transmitting coded signals downward to receivers on the Earth's surface orbiting at $55^{\circ}$ inclinations, evenly distributed in six distinct orbital planes around the globe at $20,200 \mathrm{~km}$ altitude with an orbital period of $12 \mathrm{~h}$. The continues transmitted frequencies of each satellite is on $\mathrm{L}$ band micro wave spectrum, $1575.42 \mathrm{MHz}$ (L1) and $1227.60 \mathrm{MHz}$ (L2) with two different codes P1 (or C/A) and P2 and two different carrier phases (Misra and Enge, 2006). The second is the Control Segment consists of four monitor stations and four ground antennas which are distributed around the Earth for monitoring satellites and sending signals upward for the engineering control of each 
satellite and its transmitted codes and waveforms, and the last is the user segment which includes everyone with a GPS receiver who is making use of the transmitted signals. The effect of the interaction of solar radiation with atoms and molecules in the ionosphere result in the formation of ionospheric TEC which has a diurnal variation (El rabbany, 2003) the ionization builds up during and after sunrise step by step mainly due to ultra-violet radiation and reduces rapidly at sunset hours. However during periods of instability in the atmosphere originating from external disturbances of the ionospheric region such as during ionospheric storms, the GPS-TEC moves in a wave like pattern. Such wavering can lead to errors on GPS position estimates (Olwendo, 2010 Skone, et al., 2001). The result of ionospheric effect on GPS signals thus provides data necessary for interpreting the temporal and spatial variations of the ionosphere and its effects on navigation. Total electron content is a key parameter in the mitigation of ionospheric effects on radio systems. Total electron content (TEC) is the number of electrons present per $\mathrm{m}^{2}$ along a pathway between two points. It is the integral of electron density along the ray path between ground stations and Global Positioning System (GPS) satellites, with units of electrons per square meter, where $10^{16}$ electrons $/ \mathrm{m}^{2}=1 \mathrm{TEC}$ unit (TECU). Maximum TEC usually occurs in the early afternoon and minimum TEC usually occurs just before sunrise over Nigeria (Adewale et al., 2012). The ionospheric effect on GPS signals thus provides information necessary for understanding of temporal and spatial variations of the ionosphere and its effects on navigation.

Many researchers have also worked on variation of TEC using GPS data (Wu et al., 2008 a Rama Rao, 2006 bolaji et al 2012 and Rabiu et al 2014,). Rama Rao 2006 have studied the spatial and temporal variations of the equatorial ionosphere, in Indian Sector during the low solar activity period of 2004-2005. (Rabiu et al, 2014) worked on Total electron content (TEC) measured simultaneously using Global Positioning System (GPS) ionospheric monitors installed at some locations in Nigeria.

In our study, an experiment has been carried out to investigate diurnal, monthly as well as seasonal variations of ionosphere over two equatorial stations located at Ahmadu Bello University Zaria (ABUZ) and Birnin Kebbi Polytechnic (BKFP) for the year 2012 which is the year of the highest phase of solar activity cycle 24.
MATERIALS AND METHODS

One of the significant effects is that the GPS signals traversing the ionosphere undergoes an additional delay proportional to the total electron content (TEC), which is defined as total number of free electrons in column of 1 $\mathrm{m}^{2}$ cross-sectional area along the ray path from the satellite to receiver. The total number of electrons integrated along the path from receiver to each GPS satellite is given by

$$
\mathrm{TEC}=\quad \int_{\varepsilon=t}^{s \pi t} N e \cdot d \theta
$$

Where $\mathrm{Ne}$ is electron density, GPS receivers provide both carrier phase delays $L$ and pseudo ranges $P$ of the dual frequencies. Each satellite of the GPS satellites constellation broadcasts two L-band signal at two frequencies. TEC obtained by using dual frequency (as GPS satellites broad cast two L-band signals at two frequencies) concerned the GPS stations; the GPS network is termed NIGNET (NIGerian GNSS Reference NETwork). The GPS data provide an efficient way to estimate TEC values with greater spatial and temporal coverage (Davies and Hartmann, 1997). The GPS receiver can track up to11 GPS coarse acquisition code signals at the L1 frequency of $1.575 \mathrm{GHz}$ Bolaji et al. (2012). The GPS data is in Receiver Independent Exchange (RINEX) format. RINEX is data interchange format for raw satellite navigation system data. The RINEX observation files were processed by the GPS-TEC analysis application software, developed by Gopi Seemala of the Institute for Scientific Research, Boston College, USA.

The ionosphere is a dispersive medium, hence the GPS frequencies L1 (1575.42 MHz) and L2 $(1227.6 \mathrm{MHz})$ used in the GPS system are sufficiently high, the signals are minimally affected by the ionospheric absorption and Earth's magnetic field both in the short-term as well as in the long-term changes in the ionospheric structure. There are two cases to calculate relative phase delay between the two carrier frequencies: The pseudo range method gives an absolute scale of TEC with less precision and the differential phase method, which increases measurement precision but does not allow knowing the number of cycles of phase. The ambiguity term, is resolved by combining the two estimates of TEC (Eqs. (1) and (2)) to form an improved estimate of absolute TEC (Bolaji et al., 2012).TEC arising from the group delay from pseudo range measurement can be given as (Opperman et al., 2007). 
Special Conference Edition, November, 2017

$$
T E C_{g}=\left(\frac{f_{2}^{2}-f_{1}^{2}}{f_{1}^{2} f_{2}^{2}}\right)^{-1}\left(P_{1}-P_{2}\right)
$$

Where and are $L_{1}$ and $L_{2}$ carrier frequencies, and $P_{1}$ and $P_{2}$ are pseudo-range observables, respectively. TEC from carrier phase measurement is given by

$$
T E C_{P}=\left(C_{1}-C_{2}\right) \times 2852
$$

Where $C_{1}$ and $C_{2}$ are phase measurements in Nano seconds.

Slant TEC measurements made are the sum of the real slant TEC, the GPS satellite differential delay $b_{s}$ (satellite bias) and the receiver differential delay, $b_{R}$ (receiver bias). The resultant absolute TEC is the so-called GPSderived Slant TEC (STEC) along the signal path between satellite and receiver. The Slant TEC calculated along the oblique signal paths are mapped to vertical TEC (VTEC) values by means of the Single Layer Model (SLM): the vertical TEC can be expressed as

$$
V T E C=\operatorname{STEC}-\frac{\left[b_{E}+b_{s}+b_{R K}\right]}{S(E)}
$$

Where "STEC" is the slant TEC measured, E is the elevation angle of the satellite in degrees,
$S(E)$ is the obliquity component with zenith angle $z$ at the lonospheric Pierce Point (IPP) and "VTEC" is the vertical TEC at the IPP. The obliquity factor, $S(E)$ (or the mapping function) is defined as (Bolaji et al.;2012, Olwendo et al., 2012; Langley, 1990)

$$
S(E)=\frac{1}{\cos (Z)}=\left\{\left(\frac{R_{E} \times \cos (E)}{R_{E}+h_{z}}\right)^{2}\right\}^{-(1 / 2)}
$$

where $R_{E}$ is the mean radius of the Earth in km, $h_{S}$ is the altitude of the ionosphere above the Earth's surface, $\mathrm{z}$ is the zenith angle and $\mathrm{E}$ is the elevation angle in degrees. The vertical TEC (VTEC) thus measured is used in deriving the results presented in the following sections of this paper. The estimated STEC data, S4 index, and other useful information like navigation and receiver positioning files are recorded at 1 min intervals.

RESULTS AND DISCUSSIONS

Figures 1 \& 2 show the monthly VTEC for both Zaria and Birnin-Kebbi Stations, Figures 3 \& 4 are the seasonal variation of VTEC while, Figures 5 \& 6 are the three-season variation of VTEC.

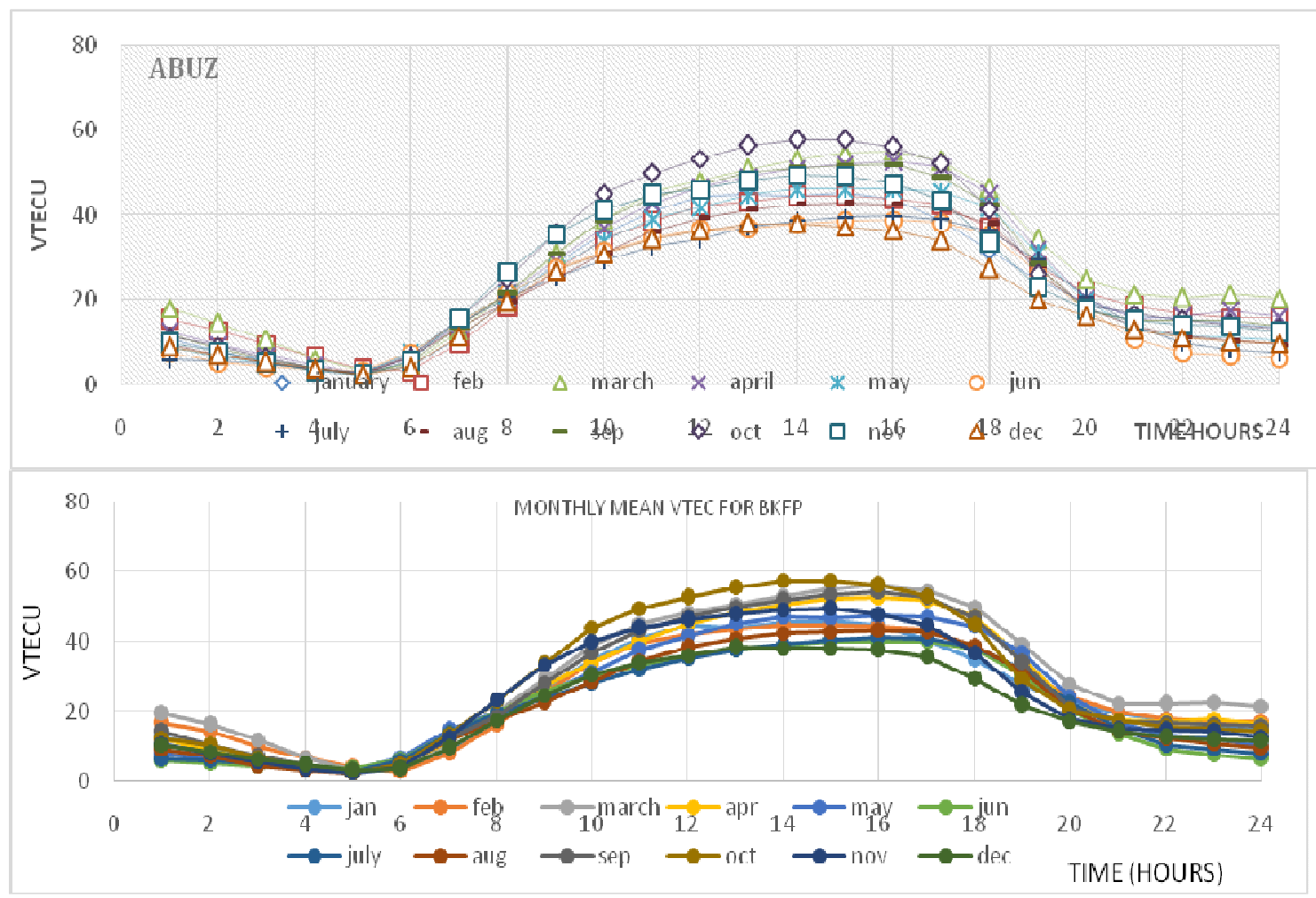

Fig.1 and 2: Monthly Mean VTEC for BirninKebbi (January - December, 2012) 


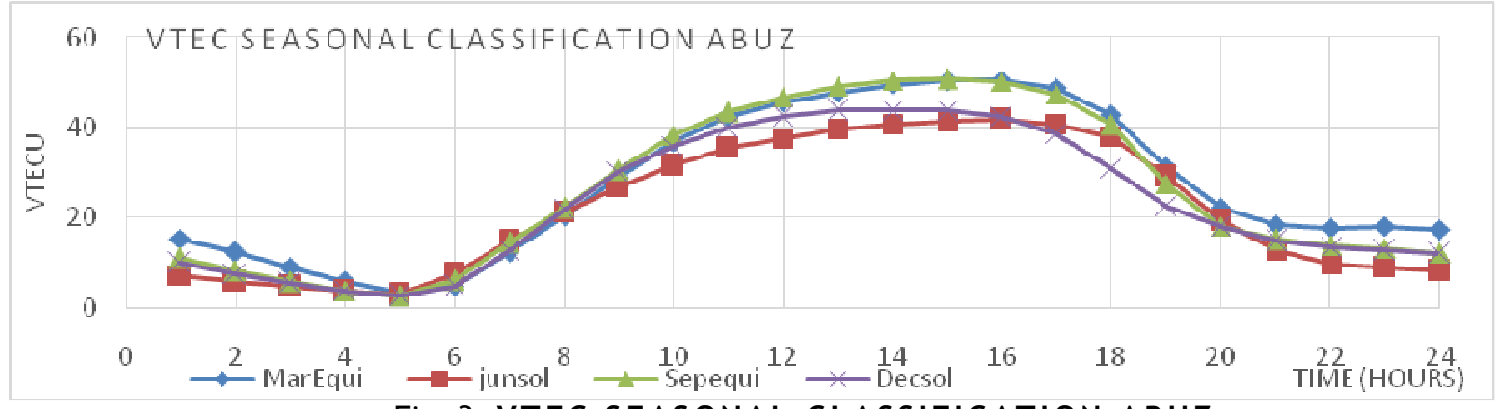

Fig. 3: VTEC SEASONAL CLASSIFICATION ABUZ

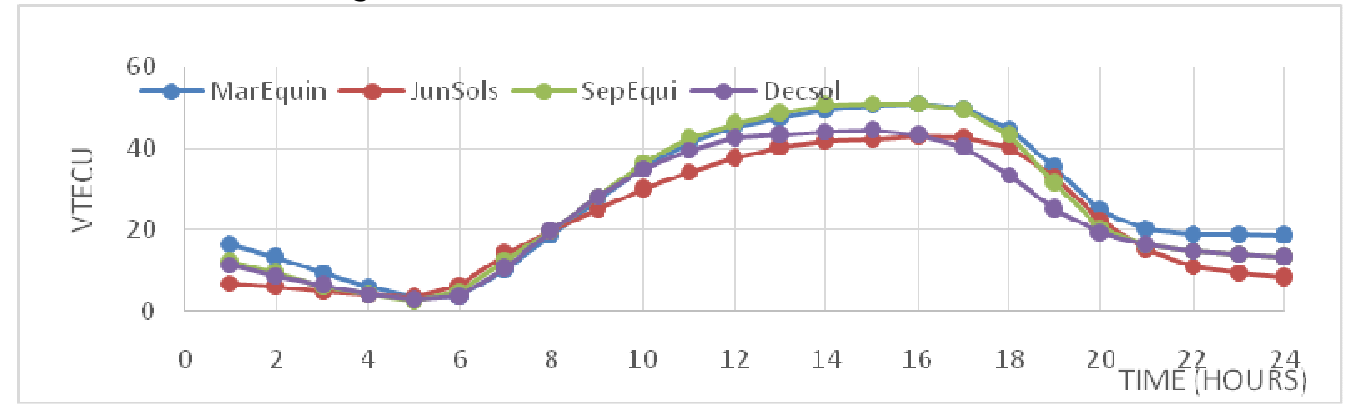

Fig. 4: VTEC SEASONAL CLASSIFICATION BKFB

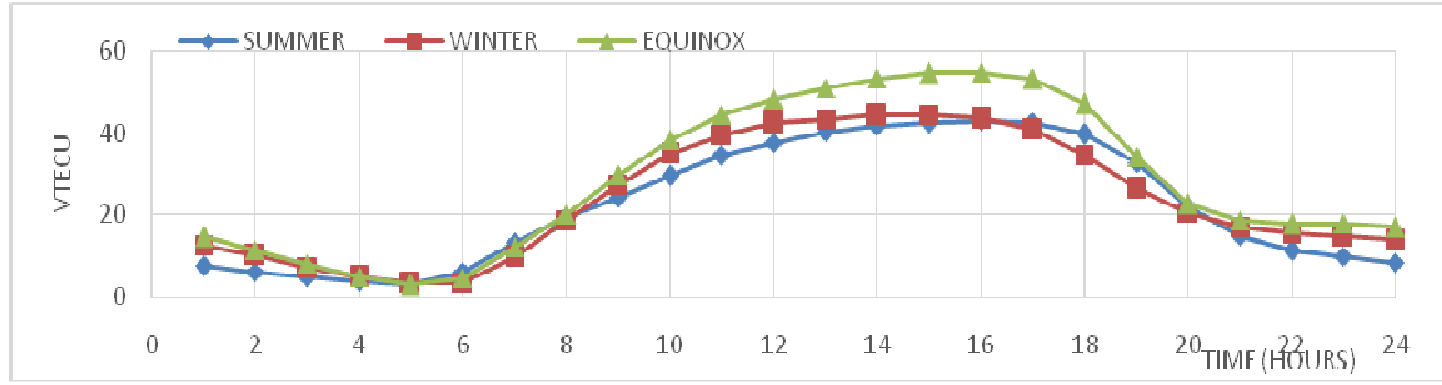

Fig.5:Three-Seasonal variation of VTEC BKFP GPS station

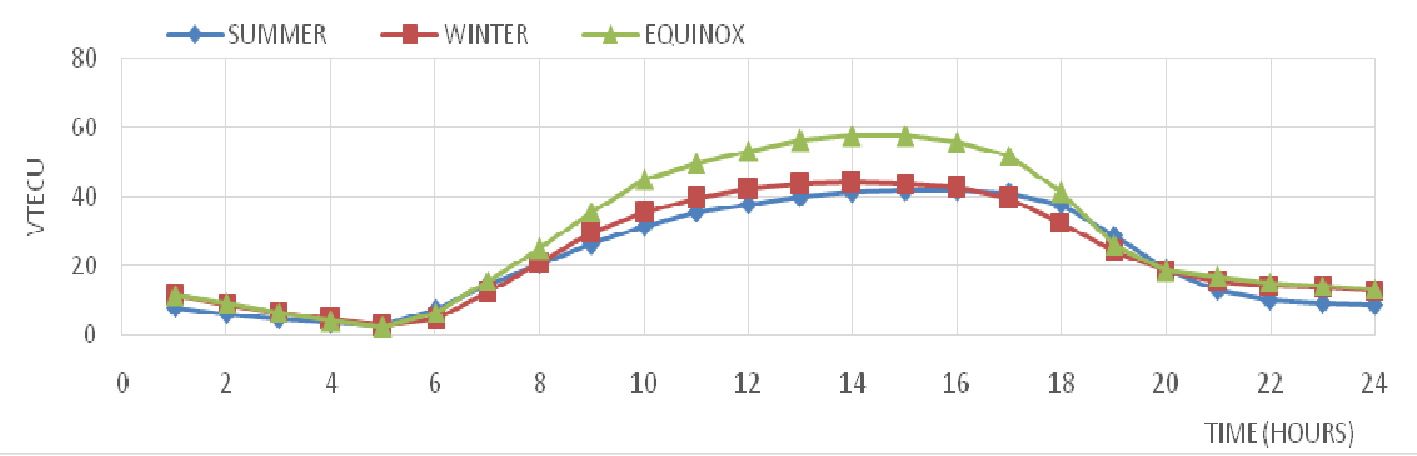

Fig.6: Seasonal variation of VTEC ABU Zaria GPS station

\section{Monthly Variation of VTEC}

Monthly variations of vertical total electron content (VTEC) have been studied by plotting the monthly mean of VTEC of the year 2012 as shown in Figures 1 and 2 respectively for the two selected stations. The plots show that VTEC has higher values during daytime compared with nighttime values, over all the stations and in all the months. The daily peak values of the VTEC occurred around 14:00 to 17:00 LT. The curves shows there is considerable day-to-day variation and month to month variations of TEC derived from different satellite passes, particularly, during the mid-day to pre-dawn hours which is a serious concern in forecasting and navigation (Rao et al., 2006). Observations at the two stations showed that the VTEC values are high in October with highest TEC of $\sim 58 \mathrm{TECU}$ occurs around 15:00LT, followed by March, September and April with highest TEC of $\sim 56, \sim 54$ and 52TECU respectively occurred around 15:00 to 16:00LT. VTEC values are low in December with highest TEC of $\sim 38 \mathrm{TECU}$, compared to the other months. 
During the daytime, the equator is hotter than the North and South poles. This causes meridional wind flows towards the poles from the equator. As reported by Balan et al. (2000), this flow of meridional wind changes the neutral composition and $\mathrm{O} / \mathrm{N}_{2}$ decreases at equatorial stations. This decrease in $\mathrm{O} / \mathrm{N}_{2}$ ratio which is maximum during the equinox months will result in higher electron density; hence, equinox VTEC will be highest as found by our result. The day-to-day variations of TEC may be attributed to the changes in solar activity which is associated with changes in the intensity of the incoming radiations (Fayose et al., 2012). The diurnal variation in TEC shows that the time at which TEC attains maximum vary from day to day. Large variations of TEC are observed in daytime while nighttime variations are found to be almost constant. VTEC values generally increase from 0600 LT for all the months and reach a maximum value during 1300-1700 LT. In addition, VTEC exhibits the usual diurnal variation of a minimum in the presunrise hours $(0500 \mathrm{LT})$ with a minimum TEC of $\sim 3 T E C U$ at all the stations and months. These diurnal monthly variations are caused by Extreme Ultraviolet flux, geomagnetic activity, equatorial electrojet and local atmospheric conditions in the thermosphere (Rabiu et al., 2014). From all the months, the magnitude range of the daytime is between $\sim 38-\sim 58$ TECU, it is clear that the VTECH for all the months during daytime is greater than the minimums range observed at nighttime (post sunset and pre sunrise) magnitude range of 5TECU to 28TECU. The solar EUV ionization coupled with the upward vertical $E \times B$ drift during the daytime give rise to this greater magnitude of VTEC during daytime. These results are in agreement with the results found at the equatorial region by (Rao et al., 2006; Bolaji et al., 2012; Olwendo et al., 2012). They concluded that the ionosphere can be lifted by upward drift velocity to higher altitude where the ionization loss rate is smaller. This phenomenon may be attributed to chemical loss of the daytime fountain effect at the magnetic equator. They further suggested that in hastening this chemical loss during daytime, regular chemical decay is increased by downward driven motions of both pole ward and zonal (eastward) neutral wind blowing away the fountain effect from the equator at noon until afternoon(Rabiu et al. 2012).

\section{Seasonal Variation}

We have used VTEC values for March Equinox (ME) (February, March, April), June Solstice (JS) (May, June, July), September Equinox (SE)
(August, September, October), and December Solstice (DS) (November, December, January), for the year 2012, with an average sunspot number $\left(R_{z}\right)$ of 57.58 to represent a period of medium solar activity (MSA).

The primary source of ionization and energy for the ionosphere is photo ionization. Therefore, The ionosphere shows strong seasonal and solar cycle flactuations (variations) on account of solar zenith angle or the solar radiations flux change, these changes may be reflected by the ionosphere dynamics. The ionosphere's seasonal variations is connected to solar zenith angle change, while its solar cycle variation is related to a change in solar EUV and X-ray radiation fluxes (Schunk and Nagy, 2009; Kelley, 1989). To investigate the seasonal variation of the ionosphere the hourly values for all days of the season were average. The mean daily hourly VTEC are obtained by averaging all the observations at a particular hour at different IPPs for all satellite in view. The mean daily hourly VTEC are then summed for all the days of the season and averaged over the number of days within the season to give the mean hourly seasonal values. It is clearly shown from the plots that VTEC has highest value in September equinox (51TECU) around 15:00LT followed by March equinox with highest TEC of 51TECU in the hours of 1600LT and the lowest magnitude of VTEC is observed in June solstice ( 43TECU) around 16:00LT in BKFP so also the same pattern is observed in ABUZ station with a highest amplitude of -51TECU early 1500LT in September equinox followed by march equinox with TEC of 51TECU occurred in the hours of $1600 \mathrm{LT}$ and lowest amplitude of $\sim 42$ TECU around 1600LT in June solstice, this mean that highest electron density is observed in equinoxes the 'winter anomaly in seasonal variation is observed. The time of occurrence of the diurnal maximum also varies with season. TEC reaches its daytime peak earlier (1400 LT) at the December solstice and later (1500 LT) at the June solstice. Since VTEC is lowest in the June solstice for both stations. This is a unique finding since as expected; it is in the equinoxes when the sub solar point is around the equator that there is high photo-ionization which produce more electrons and therefore enhancing the background electron density which results into maximum VTEC during the season (Wu et al., 2004). On the other hand during the solstices, photo-electrons at the equator decrease because the sub solar point moves to higher latitudes and other factors driving the equatorial TEC maximization such as the fountain effect are expected to decline. 
Special Conference Edition, November, 2017

Rabiu, et al. (2007) attribute the seasonal variation to the electrodynamics effect of local winds since winds are subjected to day to day and seasonal variation.

We also used a classification of seasons into summer, (May, June, July, August), winter (November, December, January February) and equinox (March, April, September, October). As recent studies (Pham et al., 2011) showed a large symmetry between the two equinoxes, this study has investigated the two equinoxes separately. This separation is also consistent with the seasons given by the zonal mean circulation of winds in the thermosphere. The winds are flowing from the summer to the winter, during summer times and reverse during winter times The values of diurnal peak in VTEC is maximum during the equinox months which occurs around 16:00LT ( 55TECU) in TEC and minimum around 05:00 to 06:00 LT ( $~ 3$ TECU) in TEC, and TEC is higher ( 45TECU) in winter than the summer ( 43 TECU) months with maximum occurrence around 15:00 00 LT in TEC minimum of ( 3 TECU) occurred at 0500 LT in BKFP. So also the same occurrence in ABUZ station with a highest TEC of 58TECU in equinox around 1400LT and the lowest TEC density is in the summer months ( 42TECU) around 1500LT.The VTEC exhibit winter anomaly this effect is caused by seasonal changes in neutral gas composition and suggested that is due to the increase in $O / N_{2}$ and the fact that the vertical wind are down ward in winter resulting in increased of $O / \mathrm{N}_{2}$ ratio and upward in summer. Consequently, recombination is weaker in the winter hemisphere, leading to higher electron

\section{REFERENCES}

Adewale, A.O., Oyeyemi, E.O., Cilliers, P.J., McKinnell, L.A., Adeloye, A.B., (2012) Low Solar activity variability and IRI 2007 predictability of equatorial Africa GPS TEC. Adv. Space Res. 49, 316-326.

Balan, N., Batista, I.S., Abdu, M.A., Bailey, G.J., Watanabe, S., MacDougall, J. and Sobral, J.H.A.(2000) Variability of additional layer in the equatorial ionosphere over Fortaleza. Geophys. Res. 105, 10603-10613.

Bolaji, O.S., A.B. Rabiu, E.O. Oyeyemi, and K. Yumoto (2012) Climatology of the interhemispheric field- aligned current $\mathrm{s}$ system over the Nigeria ionosphere. Journal of Atmospheric and SolarTerrestrial Physics 89, 144-153.

Chauhan.V and Singh, O.P. (2010) A morphological study of GPS-TEC data at concentration in the winter solstice than that in the summer solstice. Further, Earth's magnetic field is believed to guide plasma from the summer to the winter hemisphere.

\section{CONCLUSION}

This paper presents the variations of the equatorial ionosphere using the simultaneous measurements of TEC made with the GPS network of receivers on the equator. The diurnal and seasonal variations of TEC derived from all the stations are studied for a 12-month period during the medium sunspot activity (LSSA) period from January 2012 to December 2012, the results of which are summarized and presented. The results showed that VTEC has higher values during daytime when compared with nighttime values. TEC values generally increase from $0600 \mathrm{~h} \mathrm{LT}$ in all the seasons and reach its maximum value during $1500 \mathrm{~h}-1700 \mathrm{~h}$ LT. Generally, the results obtained revealed that daytime values of VTEC are greater in DECSOLS while the lowest values are observed during JUNSOLS. The day-to-day variability in TEC is also significant at all the stations, particularly during daytime hours.

\section{Acknowledgement}

We hereby express our profound gratitude to the NIGerian GNSS Reference NETwork (NIGNET) project, in the Office of the Surveyor General of Nigeria for providing us with the GPS data used in this work. Also, many thanks to Dr. Gopi K. Seemala of the Indian Institute of Geomagnetism (IIG), Navi Mumbai, India for granting us access to the GPS TEC analysis software.

Agra and theircomparison with the IRI model. www.sciencedirect.com Advances in Space Research: The official Journal of the Committee on Space Research (COSPAR) 46, 280-290. Davies, K and G. K. Hartmann (1997) Studying the ionosphere with the Global Positioning System. Radio Science 32 (4) 1695-1703

El-Rabbany, A. E (2003) Effect of Temporal Physical Correlation on Accuracy Estimation in GPS Relative Positioning. Journal of Surveying Engineering, 129 (1)

Fayose, R.S, Rabiu B, Olakunle $O$ and Keith G. (2012) Variation of Total Electron Content [TEC] and Their Effect on GNSS over Akure, Nigeria. 4 (2). doi:10.5539/apr.v4n2p105 URL: http: //dx.doi.org/10.5539/apr.v4n2p10 5 
Hong, C.-K., D. A. Grejner-Brzezinska, and J. H. Kwon (2008) Efficient GPS receiver DCB estimation for ionosphere modeling using satellite-receiver geometry changes. Earth Planets Space60(11)2528.

Kelley, M.C. (1989) The Earth's Ionosphere: Plasma Physics and Electrodynamics, International Geophysics Series, Academic Press, San Diego, CA.

Kumar, S, and Singh A.K. (2009) Variation of ionospheric total electron content in Indian low latitude region of the equatorial anomaly during May 2007April 2008 Www.sciencedirect.com. Advances in Space Research 43 (2009) 1555-1562.

Liu J, Zhao B, and Liu L. (2010) Time delay and duration of ionospheric total electron content responses to geomagnetic disturbances. Ann Geophys, 28: 795-805

Olwendo, J.O., P. Baki, P.J. Cilliers, C. Mito and P. Doherty (2012) Comparison of GPS TEC measurements with IRI2007 TEC prediction over the Kenyan region during the descending phase of solar cycle, Adv Space Res., 49, 914-921.

Opperman, B.D.L., P.J. Cilliers, L.A. McKinnel and R. Haggard (2007) Development of a regional GPSbased ionospheric TEC model for South Africa, Adv Space Res., 39, 808-815.

Oyeyemi, E. O., andA. W. V. Poole(2004)Towards the development of a new global $f_{0} F_{2} \quad$ empirical model using neural networks, Adv. Space Res., 34, 1966-1972.

Pham, H. Thi Thu, C. Amory-Mazaudier, and M. Le Huy (2011) Time variations of the ionosphere at the northern tropical crest of ionization at Phu Thuy, Vietnam. Ann. Geophys., 29, 197-207.

Rabiu, A. B., Mamukuyomi, A. I., and Joshua, E. O. (2007) Variability of equatorial ionosphere inferred from geomagnetic field measurement. Bulletin astronomy society India, 1-12.
Rabiu, A.B, A.O. Adewale, R.B. Abdulrahim, and E.O. Oyeyemi (2014) TEC derived from some GPS stations in Nigeria and comparison with the IRI 2011 and NeQuick models. Advances in Space Research 53, 1290-1303.

Rao, P.V.S., Gopi Krishna, S., Niranjan, K., and Prasad, S. V. V. D. (2006) Temporal and spatial variations in TEC using simultaneous measurements from the Indian network of receivers during the low solar activity period of 2004-2005. AnnalesGeophysicae, 24, 32793292.

Rao, G SasiBhushana, Ramu Pillalamarri and Srinivarakumar, S (2010)A Novel Approach for Efficiency Enhancement and Size Miniaturization of UWB Printed Antennas. International Journal Of Engineering-Annals Of Faculty Engineering HunedoaraRomania, Tome VIII, Fascicule 1, 67-72.

Schunk, R.W., and Nagy, A.F. (2009) lonosphere, Physics, Plasma Physics and Chemistry. Cambridge University, Press, New York, USA.

Skone, S., M. El-Gizawy and S. M. Shrestha (2001) Limitation in GPS positioning accuracies and receiver tracking performance during solar maximum. Proceeding of KIS, Banff, Canada, June 2001

Wu, C.C., Fry, C.D., Liou, K, and Tseng, C.L. (2004) Annual TEC variation in the equatorial Anomaly region during the solar minimum: September 1996-August 1997. J. Atmos. Terr. Phys. 66, 199207.

Wu, Q., T. L. Killeen, D. A. Ortland, S. C. Solomon, R. D. Gablehouse, R. M. Johnson, R. J. Niceijewski, W. R. Skinner, and S. Franke (2006) TIDI observations of migrating diurnal 